\title{
Integration of Ab Initio Nuclear Physics Calculations with Optimization Techniques
}

\author{
Masha Sosonkina ${ }^{1}$, Anurag Sharda ${ }^{1}$, Alina Negoita ${ }^{2}$, and James P. Vary ${ }^{2}$ \\ ${ }^{1}$ Ames Laboratory/DOE, Iowa State University, Ames, IA 50011, USA \\ \{masha, anurag\}@scl. ameslab.gov \\ ${ }^{2}$ Physics Department, Iowa State University, Ames, IA 50011, USA \\ \{alina, jvary\}@iastate.edu
}

\begin{abstract}
Optimization techniques are finding their inroads into the field of nuclear physics calculations where the objective functions are very complex and computationally intensive. A vast space of parameters needs searching to obtain a good match between theoretical (computed) and experimental observables, such as energy levels and spectra. In this paper, we propose a design integrating the ab initio nuclear physics code MFDn and the VTDIRECT95 code for derivative-free optimization. We experiment with the initial implementation of the design showing good matches for several single-nucleus cases. For the parallel MFDn code, we determine appropriate processor numbers to execute efficiently a multiple-nuclei parameter search.
\end{abstract}

Keywords: No Core Shell Model, MFDn, Derivative-free Optimization, VTDIRECT95.

\section{Motivation}

Unlike electrons in the atom the interaction between nucleons is not known precisely and is complicated. The shell model is the fundamental tool to study the structure of nuclei. The basic idea is that the nucleons move in an average potential generated by the mutual interactions of the nucleons. The strong Nucleon-Nucleon (NN) interaction as well as 3-nucleon (NNN) interactions generate the potential that describes the nucleon energy levels in the nucleus. In particular, NN and NNN interactions tuned to fit light nuclei are used in nuclear astrophysics for solar models, supernova modeling, and Big Bang nucleosynthesis. The techniques for solving these problems also find applications in the field of quantum chemistry, condensed matter physics, atomic, nuclear, and particle physics.

Until recently, the No Core Shell Model 112 has been limited to nuclei up to atomic mass $A$ of 16 . Work is underway to extend this method to heavier nuclei 3. The effective Hamiltonian operator derived from CD-Bonn interaction [4] gives a poor description of nuclei with atomic mass near 48. Figure 1 describes the matches between the theoretical and experimentally obtained energy levels for ${ }^{49} \mathrm{Sc}$, where the initial version of the theory is given in the rightmost column.

M. Bubak et al. (Eds.): ICCS 2008, Part I, LNCS 5101, pp. 833 842, 2008.

(C) Springer-Verlag Berlin Heidelberg 2008 
A problem with the existing Hamiltonian is that the computed spectra is too compressed compared with the experimental spectra. The addition of the three terms - isospin-dependent $V_{0}$, central $V_{1}$, and tensor-interaction $V_{\text {tens }}$ - results in a reasonable low lying spectra for the nuclei involved in the double-beta decay of ${ }^{48} \mathrm{Ca}$. One of the physics goals is to test whether the same modified Hamiltonian used for the isotopes of the nuclei with atomic mass of 48 is able to describe other heavy nuclei. These three terms and possibly other (up to 20) parameters need to be searched to obtain their best match to the experimental values (see Fig. 1, for example). To find this match according to some criteria, it is required to evaluate energies at many points in a parameter search space. In particular, a criterion, called $\chi^{2}$, may be calculated that quantifies the match using weights (see Sect. 3.2). This process may be automated by taking advantage of optimization techniques which will generate the points at which $\chi^{2}$ may be evaluated. Note that, since derivatives do not come into the picture for complex nuclear physics calculations on which $\chi^{2}$ depends, the derivative-free optimization is considered. As a trade-off, it is typically required a large number of function evaluations even to find a local minimum. The time taken by such an optimization algorithm is directly proportional to the cost of the objective function evaluation. Thus, parallel implementations of both the function evaluation and optimization algorithm may be beneficial.

The remainder of the paper is organized as follows. In Sect. 2] we give an overview of two parallel software packages under consideration: Many Fermion Dynamics nuclear (MFDn) and the optimization algorithm VTDIRECT95. In

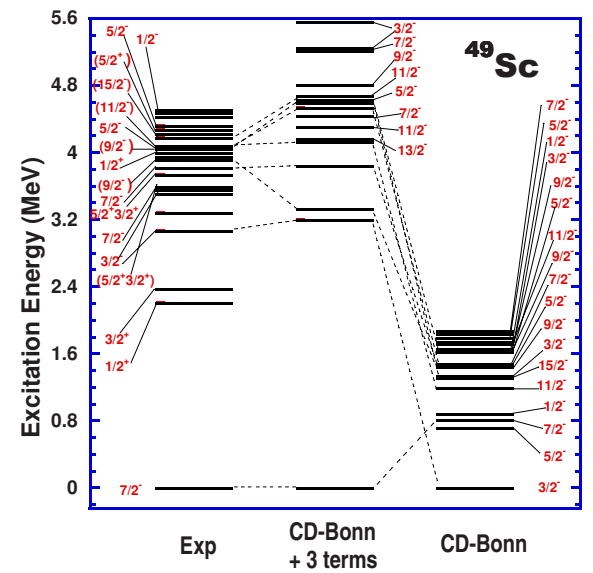

Fig. 1. Matching of experimental (Exp) and theoretical energy levels of ${ }^{49} \mathrm{Sc}$ using CD-Bonn potential in its initial version (CD-Bonn) and new with searched terms (CD-Bonn+3terms). Each energy level is annotated with its spin value.

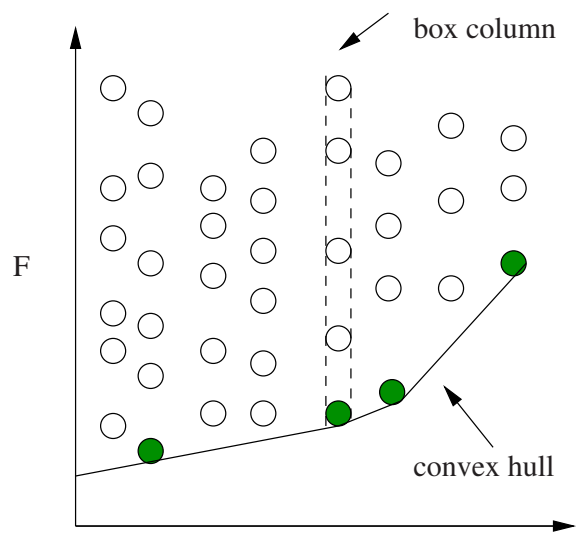

D

Fig. 2. Example of the scatter plot of boxes. The $F$-axis is function values, and the $D$-axis is box diameters. 
Sect. 3. we first present a design to make the MFDn code and optimization algorithm work in concert. Then, we describe our initial implementation. Sect. 4 presents the computation experiments and analyzes them with respect to nuclear physics objectives. Sect. 5 concludes.

\section{Overview of Nuclear Physics and Optimization Packages}

Many Fermion Dynamics nuclear (MFDn) 5] parallel code is used for large-scale nuclear structure calculations in the No Core Shell Model (NCSM) formalism [1/2], which has been shown to be successful for up to 16-nucleon problems on present day computational resources. MFDn code is charged to compute a few lowest $(\approx 15)$ converged solutions, called wave functions, to the many-nucleon Schrödinger equation:

$$
H|\phi\rangle=E|\phi\rangle .
$$

Then other properties, called observables, are formed from the calculated wave functions. The matrix $H$ in (1) is the Hamiltonian operator, which is typically solved using Lanczos diagonalization since $H$ is symmetric and sparse. However, the Lanczos iterative process may be very expensive due to huge dimensionality of $H$ with many off-diagonal elements. The number of Lanczos iterations also increases significantly for the energy levels beyond the ground state. For example, for the ${ }^{16} \mathrm{O}$ nucleus in the $6 \bar{h} \omega$ basis space, the ground-state energy level requires only 35 Lanczos iterations, while 15 excited states need at least 200 Lanczos iterations to converge. Note that, in this case, the constructed Hamiltonian $H$ has the dimension of 26,483,625. MFDn constructs the $m$-scheme basis space, evaluates the Hamiltonian matrix elements in this basis using efficient algorithms, diagonalizes the Hamiltonian to obtain the lowest eigenvectors and eigenvalues, then post-processes the wave functions to obtain a suite of observables and to compare them with experimental values.

VTDIRECT95 6] is a Fortran95 suite of parallel codes implementing the derivative-free optimization algorithm DIRECT [7] that takes a set of problemand algorithm-dependent input parameters and finds the global minimum of an objective function $f$ inside the feasible set $D$. Each iteration of DIRECT consists of the following steps.

1. INITIALIZATION. Normalize the feasible set $D$ to be the unit hypercube. Sample the center point $c_{i}$ of this hypercube and evaluate $f\left(c_{i}\right)$. Initialize $f_{\min }=f\left(c_{i}\right)$, evaluation counter $m=1$, and iteration counter $t=0$.

2. SELECTION. Identify the set $S$ of "potentially optimal" boxes that are subregions of $D$. A box is potentially optimal if, for some Lipschitz constant, the function value within the box is potentially smaller than that in any other box (a formal definition with parameter $\epsilon$ is given by [8]).

3. SAMPLING. For any box $j \in S$, identify the set $I$ of dimensions with the maximum side length. Let $\delta$ equal one-third of this maximum side length. Sample the function at the points $c \pm \delta e_{i}$ for all $i \in I$, where $c$ is the center of the box and $e_{i}$ is the $i$ th unit vector. 
4. DIVISION. Divide the box $j$ containing $c$ into thirds along the dimensions in $I$, starting with the dimension with the lowest value of $w_{i}=\min \{f(c+$ $\left.\left.\delta e_{i}\right), f\left(c-\delta e_{i}\right)\right\}$, and continuing to the dimension with the highest $w_{i}$. Update $f_{\min }$ and $m$.

5. ITERATION. Set $S=S-\{j\}$. If $S \neq \emptyset$ go to 3 .

6. TERMINATION. Set $t=t+1$. If iteration limit or evaluation limit has been reached, stop. Otherwise, go to 2.

Initially, only one box exists in the system. As the search progresses, more boxes are generated, illustrated by the scatter plot shown in Fig. 2, where each circle represents a box. The sizes of boxes increase along the $D$-axis (diameter) and the function values at box centers increase along the $F$-axis (function). All the boxes with the same diameter belong to a "box column". Reference 8] proves that all potentially optimal boxes in $S$ are on the lower right convex hull of the scatter plot in Fig. 2. To produce more tasks in parallel, new points are sampled around all boxes in $S$ along their longest dimensions during SAMPLING. This modification also removes the step ITERATION, thus simplifying the loop. In the DIVISION step, multiple new boxes are generated for each potentially optimal box. The multiple function evaluation tasks at each iteration give rise to a natural functional parallelism, which is especially beneficial for expensive objective functions. The parallel implementation distributes the work to multiple masters in the SELECTION phase. The functions are then evaluated by the pool of workers to accomplish SAMPLING. VTDIRECT95 also supports user-level checkpointing method to restart function evaluations through log files. Several other options are provided by the optimization algorithm to improve the performance on large-scale parallel systems.

\section{Design of Integrated System}

In nuclear structure calculations, the computed and experimental results are matched for single as well as multiple nuclei using the $\chi^{2}$ function. Thus, a general case of multiple nuclei is considered in the design. At the initialization, the search algorithm divides the search domain into multiple sub-domains and assigns each sub-domain a set of masters (SM). Each sub-domain master is responsible for generation of evaluation points within its own sub-domain. Figure 3 shows a diagram for the case of multiple nuclei, N1, N2, and N3, with one MFDn execution per nuclei. The overall hierarchy consists of three tiers. The first two tiers correspond to the set of processors used by VTDIRECT95 while the last tier corresponds to the set of processors used by MFDn. It is denoted as MFDn pool and included into a dash-lined oval. The evaluation points generated by SM are then handed to the workers W assigned to VTDIRECT95 (represented as shaded squares in Fig. 3). These workers act as connectors between the VTDIRECT95 and the MFDn. Each worker W pre-processes the evaluation point and then delivers the pre-processed data to MFDn pool. The number of processing elements (PE) required by MFDn typically depends on the size of the Hamiltonian matrix and the hardware characteristics. So another functionality of a $\mathrm{W}$ 


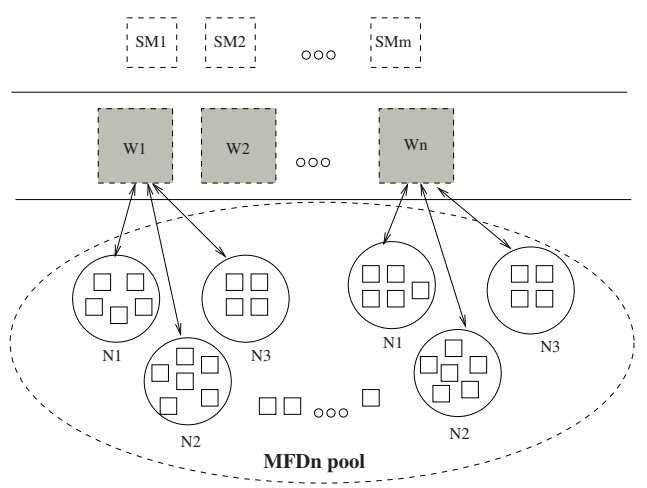

Fig. 3. Design layout for the integration system

worker is to assemble from MFDn pool the needed number of PEs for an MFDn run. Once an instance of MFDn completes, its output is communicated back to the associated $\mathrm{W}$ worker, which is responsible for computing the $\chi^{2}$ function aggregating the MFDn outputs for all the nuclei and for delivering it to the proper SM.

\subsection{Enabling Seamless Integration}

The goal of this design is to treat both the VTDIRECT95 and the MFDn codes as "black-box" components and provide an infrastructure to integrate them. Here, we detail the implementation of the design. The MFDn code requires an input file which contains the set of parameters for every MFDn run. The output of the MFDn code is a text file including theoretical observables, such as excitation energies for a given nucleus for the desired number of states. The optimization algorithm produces points in a search space in each iteration and then accepts the function evaluation at those points. Thus, both MFDn and VTDIRECT95 have well-defined input and output interfaces. Consider the following external additions necessary to interface MFDn and VTDIRECT95:

1. Input Modifier (IM) inserts the points generated by VTDIRECT95 into an input file for MFDn.

2. Wait (W) waits for a completion signal from the MFDn code. The Wait stub may also gather the runtime performance information from MFDn.

3. Output Modifier (OM) post-processes the MFDn output file and creates an input for the $\chi^{2}$ function.

For a single iteration of VTDIRECT95, Fig. 4 shows a workflow between the VTDIRECT95 and MFDn codes. The main goal of the described additions is to provide interfaces and placeholders for pre- and post-processing. Thus, we denote them as stubs in Fig. 4. VTDIRECT95 generates an evaluation point which is inserted into MFDn input file through the IM stub. MFDn produces 
then a set of values to be captured by the OM stub and provided to the $\chi^{2}$ evaluator, which in turn returns the objective function value to VTDIRECT95.

All the stubs related to the MFDn execution are grouped in a single box together with MFDn in Fig. 4. The $\chi^{2}$ evaluator is a generic module the implementation of which may change depending on the application scientist's goals. Similarly, VTDIRECT95 is depicted as separate box since it is a general-purpose optimization code. The integrated workflow, due to its flexibility, allows to change the objective function construction and the search algorithm without affecting the overall organization.

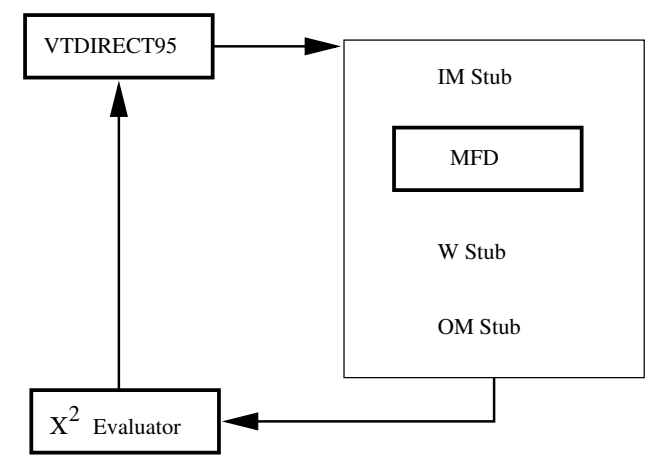

Fig. 4. Workflow diagram for the MFDn and VTDIRECT95

\subsection{Construction of the $\chi^{2}$ Function}

The $\chi^{2}$ is constructed using a theory file, an experimental file, and the base energy value of the given nucleus. The theory file is an output from the MFDn code which contains calculated observables. The experimental file has the energy levels as found experimentally by different national scientific organizations 910. In addition to the energy values, each energy level is associated with the $\operatorname{spin} j$ of the protons/neutrons. There are many options in construction of $\chi^{2}$. A particular choice depends on such parameters as the quality of the experimental data and the questions nuclear physicists want to answer comparing the theoretical and experimental energy levels. As an example consider the following $\chi^{2}$ definition:

$$
\chi^{2}(\mathbf{v})=\sum_{\substack{1 \leq i_{t} \leq 15 \\ 1 \leq i_{e} \leq k}}\left[E_{i_{e}}(\mathbf{v})-\tilde{E}_{i_{t}}(\mathbf{v})\right]^{2} \times \sigma_{i_{e}}^{2},
$$

where $\mathbf{v}=\left(V_{0}, V_{1}, V_{\text {tens }}\right)$ and $\tilde{E}$ are the absolute experimental and theoretical energies, respectively; $i_{e}$ and $i_{t}$ are the indices of the corresponding matched energy levels (one $i_{e}$ paired with one $i_{t}$ ), i.e., those levels that have the same spin $j$; and $k$ is the maximum desired number of matches. Each experimental energy level $l_{e}$ is assigned the weight $\sigma_{l_{e}}$. This weight is inversely proportional to the distance of that energy level from the ground energy level. In (2), different weight schemes were considered, such as, for every $l_{e}, \sigma_{l_{e}}=1 / l_{e}$ or $\sigma_{l_{e}}=1 / 2^{l_{e}}$. 


\section{Experiments}

Computing platforms at the National Energy Research Scientific Computing Center (NERSC) at the Lawrence Berkeley National Laboratory served as testbed for the development and testing. The results presented here were obtained on the NERSC IBM p575 POWER 5 system, named Bassi. It is a distributed memory computer with 888 processors (comprising 111 nodes and sharing 32 GBytes of memory) used for scientific applications. Each Bassi processor has a theoretical peak performance of 7.6 GFlops, and the nodes are interconnected by the IBM "Federation" HPS switch. An implementation of the proposed design for single and multiple nuclei has been developed and tested. It integrates the serial VTDIRECT95 and parallel MFDn codes, while the usage of parallel VTDIRECT95 has been left as a future work.

The following three nuclei have been considered: ${ }^{47} \mathrm{~K},{ }^{48} \mathrm{Ca}$, and ${ }^{49} \mathrm{Ca}$. The Hamiltonian matrices are sparse and their sizes are 136231, 12000, and 15666, respectively, in the lowest available model space. The MFDn execution time depends heavily on the Hamiltonian size. Also, the complexity ("shape") of the objective function drives the time required by the optimization algorithm to find the minimum. Figure 5(a) shows the number of evaluations required per VTDIRECT95 iteration as the number of iterations grows for a sample nucleus.

For the multiple nuclei fit, the runtime is guided by the runtime of the heaviest nucleus since the evaluations for all the nuclei are needed to construct the $\chi^{2}$ in this case. Therefore, it is desirable to adjust the number of processors allocated to a particular nucleus based on its computational cost relative to the other nuclei in the set. In particular, for our example, ${ }^{47} K$ has the largest Hamiltonian matrix, so executing it on the largest subset of processors makes sense. Figure 5(b) depicts the case when all the three nuclei are evaluated once with three different sets of processor numbers (shown in the $x$-axis). The parallel time to compute all three nuclei indeed decreases as the number of processors is increased compared with the base case of using small equal number of processor for each nucleus. When about twice as many (15) processors are assigned to the calculation of each nucleus, the timings for the smaller Hamiltonians decrease by about half. The runtime for ${ }^{47} K$, however, decreases only slightly. By augmenting the number of processors to 45 for ${ }^{47} \mathrm{~K}$, the execution time is decreased dramatically, while for the smallest Hamiltonian of ${ }^{48} \mathrm{Ca}$, the time has actually increased with the increase in the number of processors to 21 . The latter indicates that the parallel overhead starts to dominate the overall execution time. In general, besides the Hamiltonian matrix size, other factors such as communication overhead and hardware characteristics, may affect the number of processors used to calculate efficiently a particular nucleus.

The optimization of the parameters for ${ }^{48} \mathrm{Ca}$ produced the results (Fig. [6(a)) matching theoretical observables correctly to their counterparts in the experimental data file. First six states have been matched correctly with their spins and the difference in energy levels from theoretical observables and experimental data is less than $0.001 \mathrm{MeV}$. The second nucleus considered is ${ }^{49} \mathrm{Ca}$. The groundstate level in the theoretical observable was matched within the difference of 


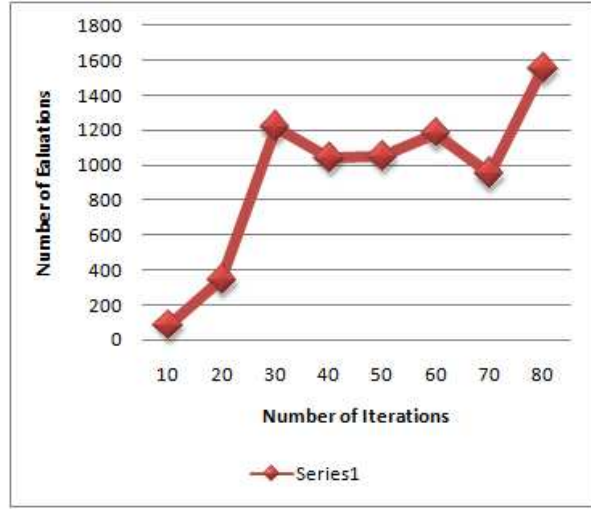

(a) Evaluations per iteration for ${ }^{49} \mathrm{Ca}$.

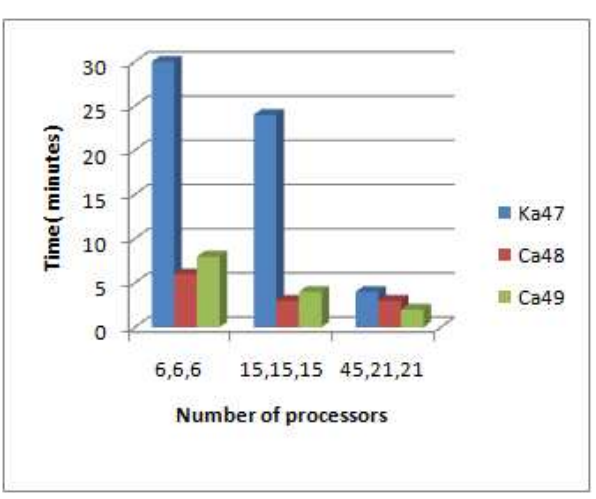

(b) Execution time of 1 evaluation of VTDIRECT95 for ${ }^{47} \mathrm{~K},{ }^{48} \mathrm{Ca}$, and ${ }^{49} \mathrm{Ca}$ on different numbers of processors on Bassi.

Fig. 5. Execution of MFDn for a single- and multiple-nuclei cases

$0.02 \mathrm{MeV}$ of the experimental ground state energy value (Fig. 6(b)). Higher energy levels in the theoretical observables remained unmatched to counterparts in the experimental data. A likely reason is that some of the energy levels in the experimental data have uncertain spin levels. The findings for ${ }^{49} \mathrm{Ca}$ are important from the physics point of view since they give new directions for fitting the nuclei with a similar mass starting with obtained set of parameters. Similar arguments may be used to explain the unmatched energy levels in ${ }^{47} K$.

Checkpointing is a valuable feature of VTDIRECT95. Supercomputers with batch scheduling typically have an upper bound on the time any job is allowed to execute. For example, the maximum time permitted on NERSC supercomputers

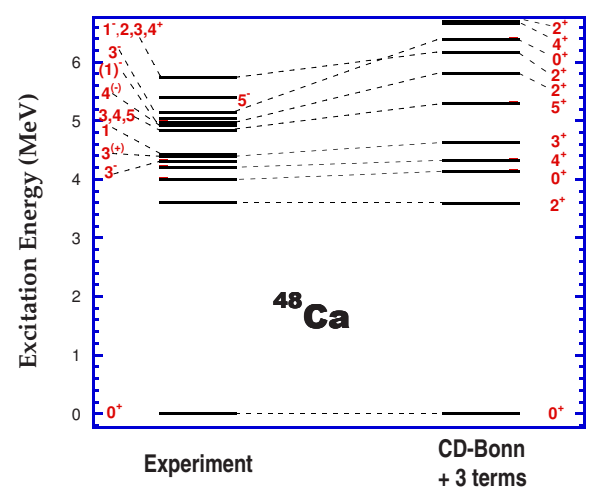

(a) ${ }^{48} \mathrm{Ca}$.

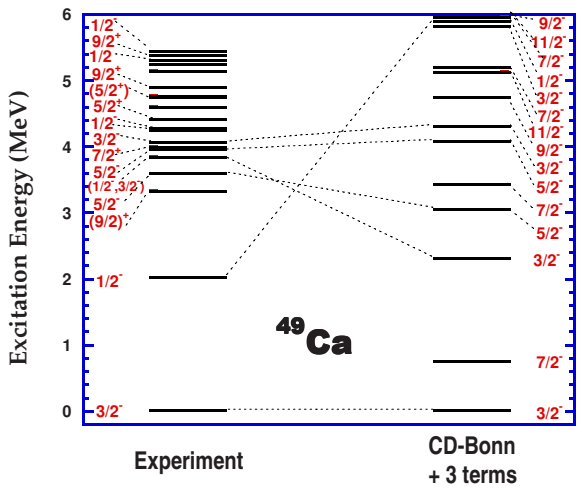

(b) ${ }^{49} \mathrm{Ca}$.

Fig. 6. Matching of experimental and theoretical energy levels 
is only 48 hours, which is surely not enough to find the global or even local minimum for such an expensive function evaluation as described in this paper. Hence, we have utilized the checkpointing feature as a routine procedure to restart the integrated code for the next maximum time allowed by the queuing system.

\section{$5 \quad$ Summary and Future Work}

We have proposed a design for the integration of the MFDn and VTDIRECT95 parallel codes. The integration uses the master-worker paradigm of the VTDIRECT95 code and produces a three-tier scheme. Our contribution is to show how an expensive multiprocessor function evaluation may fit into this scheme.

In the paper, we have shown an implementation of the proposed design for the case of sequential VTDIRECT95, which produces one point at a time. In this situation, we have studied various definitions of the objective function $\left(\chi^{2}\right)$ and obtained good matches between the theoretical and experimental energy levels for ${ }^{48} \mathrm{Ca}$ and the ground-state energy level for ${ }^{49} \mathrm{Ca}$. We have also investigated the efficient executions of MFDn when the results from multiple nuclei are to be considered simultaneously by VTDIRECT95. We have found that assigning different numbers of processors to different MFDn executions, typically in accordance with the Hamiltonian matrix size, reduces the overall time for a function evaluation needed by VTDIRECT95. In the future, we plan to provide an implementation taking advantage of parallelism in both VTDIRECT95 and MFDn and also to consider a variety of nuclei in the multiple-nuclei case. We will also compare the results produced by VTDIRECT95 with other derivative-free optimization techniques, such as those from the Toolkit for Advanced Optimization (TAO) 11. The integrated software will be a useful tool for a wide range of $a b$ initio nuclear physics calculations.

Acknowledgments. The work was supported in part by Iowa State University under the contract DE-AC02-07CH11358 with the U.S. Department of Energy, by the U.S. Department of Energy under the grants DE-FC02-07ER41457 (UNEDF SciDAC-2) and DE-FG02-87ER40371 (Division of Nuclear Physics), and by NERSC.

\section{References}

1. Navratil, P., Vary, J., Barrett, B.: Properties of ${ }^{12} \mathrm{C}$ in the ab-initio Nuclear Shell Model. Phys. Rev. Lett. 84, 5728 (2000)

2. Navratil, P., Vary, J., Barrett, B.: Large-basis ab-initio No-core Shell Model and its application to ${ }^{12} \mathrm{C}$. Physical Review C62, 54311 (2000)

3. Vary, J., Popescu, S., Stocia, S., Navratil, P.: No Core Shell Model A=47 and $\mathrm{A}=49$. nucl-th/0607041/

4. Machleidt, R., Sammarruca, F., Song, Y.: Nonlocal nature of the nuclear force and its impact on nuclear structure. Phys. Rev. C53, C63, 024001, Ref 9 (1996, 2001) 
5. Vary, J.: The Many-Fermion Dynamics Shell-Model Code. Iowa State University (1992)

6. He, J., Watson, L., Sosonkina, M.: Algorithm XXX: VTDIRECT95: Serial and parallel codes for the global optimization algorithm DIRECT. ACM Transactions on Math. Soft. (submitted, 2007)

7. Jones, D.: The DIRECT global optimization algorithm. Encyclopedia of Optimization 1, 431-440 (2001)

8. Jones, D., Pertunen, C., Stuckman, B.: Lipschtzian optimization without the Lipscitz constant. J. Optimization Theory and Applications 79, 157-181 (1993)

9. Electronic version of nuclear data sheets. telnet://bnlnd2.dne.bnl.gov

10. Burrows, T.: Nuclear data sheets, 74, 1; Nuclear data sheets 76, 191 (1995)

11. Benson, S., McInnes, L.C., Moré, J., Munson, T., Sarich, J.: TAO user manual (revision 1.9). Technical Report ANL/MCS-TM-242, Mathematics and Computer Science Division, Argonne National Laboratory (2007),

http://www.mcs.anl.gov/tao 